\title{
Postoperative complications are associated with worse survival after laparoscopic surgery for non-metastatic colorectal cancer - interim analysis of 3-year overall survival
}

\author{
Michał Nowakowski ${ }^{1}$, Magdalena Pisarska ${ }^{2,3}$, Mateusz Rubinkiewicz ${ }^{2}$, Grzegorz Torbicz ${ }^{2}$, Natalia Gajewska², \\ Magdalena Mizera², Piotr Major ${ }^{2,3}$, Paweł Potocki ${ }^{4}$, Dorota Radkowiak², Michał Pędziwiatr ${ }^{2,3}$ \\ ${ }^{1}$ Department of Medical Education, Jagiellonian University Medical College, Krakow, Poland \\ ${ }^{2} 2^{\text {nd }}$ Department of General Surgery, Jagiellonian University Medical College, Krakow, Poland \\ ${ }^{3}$ Centre for Research, Training and Innovation in Surgery, Krakow, Poland \\ ${ }^{4}$ Department of Oncology, Jagiellonian University Medical College, Krakow, Poland
}

\begin{abstract}
Introduction: Postoperative morbidity after colorectal resections for cancer remains a significant problem. Data on the influence of complications on survival after laparoscopic colorectal resection are still limited.

Aim: To analyze the impact of postoperative complications on long-term survival after radical laparoscopic resection for colorectal cancer.

Material and methods: Two hundred and sixty-five consecutive non-metastatic colorectal cancer patients undergoing laparoscopic colorectal resection for cancer were included in the analysis. The entire study group was divided into two subgroups based on the occurrence of postoperative complications. Group 1 included patients without postoperative morbidity and group 2 included patients with complications. The primary outcome was overall survival. Results: Median follow-up was 45 (IQR: 34-55) months. Group 1 consisted of 187 (70.5\%) patients and group 2 comprised 78 (29.5\%) patients. Studied groups were comparable in terms of sex, age, body mass index, ASA class, cancer staging, localization of the tumor and operative time. Patients in group 1 had significantly better overall 3-year survival compared to those with complications ( $84.9 \%$ vs. $69.8 \%, p=0.022)$. Kaplan-Meier curves showed significantly improved survival rates in patients without complications compared with complicated cases. The Cox proportional multivariate model showed that postoperative complications ( $H R=2.83 ; 95 \% \mathrm{Cl}: 1.35-5.92 ; p=0.0058)$ and AJCC III $(H R=3.17$; $95 \%$ Cl: 1.52-6.6; $p=0.0021$ ) were independent predictors of worse survival after laparoscopic colorectal cancer surgery. Conclusions: Our analysis of interim results after 3 years confirms that complications after laparoscopic colorectal cancer surgery have an impact on survival. For this reason, these patients should be carefully monitored after surgery aiming at early detection of recurrence.
\end{abstract}

Key words: laparoscopy, colorectal cancer, survival, postoperative complications.

\section{Introduction}

Postoperative morbidity after colorectal resections for cancer remains a significant problem. In spite of evolution in operative technique, implemen- tation of modern perioperative protocols and improvements in surgical instruments, postoperative complication rates range from $18 \%$ to $38 \%$ [1-5]. The short-term consequences of postoperative mor- 
bidity are well established: prolonged hospital stay, need for surgical reintervention, need for ICU stay and increased mortality [6]. This is also accompanied by higher costs of treatment [7]. Moreover, it has been shown that postoperative complications may delay chemotherapy, which influences the quality of oncologic management [8-10]. There are also reports showing that postoperative morbidity might affect long-term survival [11]. However, the majority of them include patients undergoing open or a mix of open and laparoscopic surgery [12]. Data on the influence of complications on survival after laparoscopic colorectal resection are still limited.

\section{Aim}

The aim of our study was to analyze the impact of postoperative complications on long-term survival after radical laparoscopic resection for colorectal cancer.

\section{Material and methods}

Consecutive colorectal cancer patients undergoing laparoscopic colorectal resection for cancer between 2013 and 2016 were included in the prospectively collected database. Inclusion criteria for our study were: age over 18 years, histopathologically confirmed adenocarcinoma of the colon or rectum, laparoscopic approach, and patients with at least 2-year follow-up. Exclusion criteria included emergency or initially open surgery, transanal endoscopic microsurgery, concomitant inflammatory bowel diseases, patients with stage IV cancer according to AJCC classification diagnosed before primary surgery, and patients who died within 30 days of surgery. The standard surgical procedure was laparoscopic resection combined with the well-established ERAS protocol [13-15]. When appropriate, patients with rectal cancer underwent preoperative radio- or chemoradiotherapy. All patients were discussed postoperatively during a multidisciplinary team meeting and adjuvant chemotherapy was administered according to the most recent guidelines. Figure 1 presents the patients' flow through the study.

\section{Outcome measures}

The entire study group was divided into two subgroups based on the occurrence of postoperative complications. Group 1 included patients with-

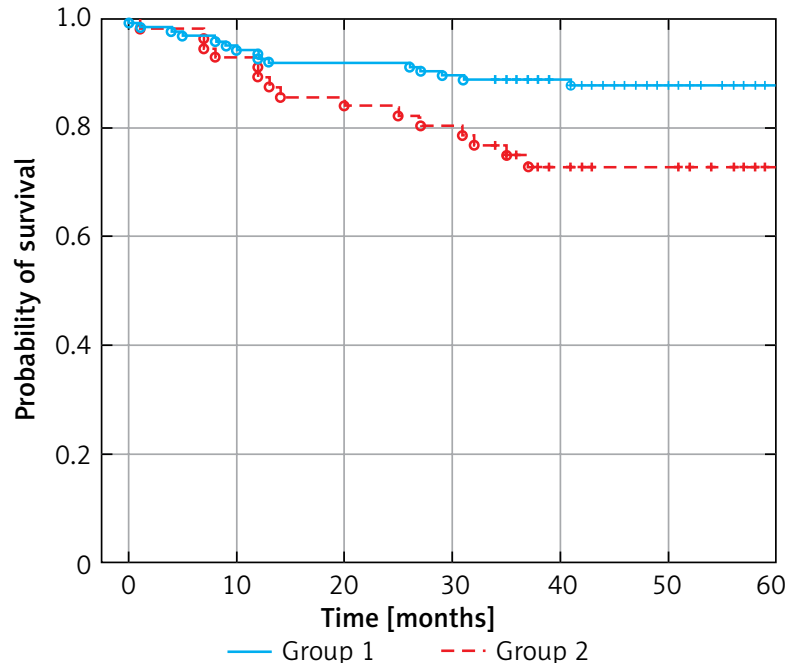

Figure 1. Kaplan-Meier curves of overall survival according to presence of complications

out postoperative morbidity and group 2 included patients with complications. They were graded according to the Clavien-Dindo classification [16, 17]. When a patient developed more than one complication, the most severe one was used for analysis. The primary outcome was overall survival. Other outcomes included the following measures: differences in survival according to severity of complications and stage of cancer.

\section{Material}

The study group included 265 patients with median 45 (IQR: 34-55) months follow-up. Group 1 consisted of 187 (70.5\%) patients without complications and group 2 comprised 78 (29.5\%) patients with at least one complication. Table 1 presents their severity according to the Clavien-Dindo classification. There were no differences between groups in terms of basic demographic parameters: sex, age, body mass index (BMI), ASA class. There was also no difference in type of surgery (colon/rectum) or operative time (median: $180 \mathrm{~min}$ ). However, we noted a statistically significant difference in intraoperative blood loss (50 vs. $100 \mathrm{ml}$, $p=0.0352$ ). Cancer staging was not different between the groups (Table I).

\section{Ethical approval}

The study was approved by the local Ethics Review Committee (approval number 1072.6120.225.2017). All procedures were performed in accordance with 
Table I. Demographic analysis of patient groups

\begin{tabular}{|c|c|c|c|}
\hline Parameter & $\begin{array}{c}\text { Group } 1 \\
\text { Non-complicated }\end{array}$ & $\begin{array}{c}\text { Group } 2 \\
\text { Complicated }\end{array}$ & $P$-value \\
\hline \multicolumn{4}{|l|}{ Basic demographic parameters: } \\
\hline Number of patients, $n(\%)$ & $187(70.6)$ & $78(29.4)$ & - \\
\hline Females, $n(\%)$ & $93(49.7)$ & $34(43.6)$ & 0.3617 \\
\hline Males, $n(\%)$ & $94(50.3)$ & $44(56.4)$ & \\
\hline Age, mean \pm SD [years] & $65.3 \pm 13.4$ & $64.1 \pm 13.1$ & 0.4269 \\
\hline $\mathrm{BMI}$, mean $\pm \mathrm{SD}\left[\mathrm{kg} / \mathrm{m}^{2}\right]$ & $26.6 \pm 4.9$ & $26.1 \pm 4.3$ & 0.7455 \\
\hline ASA 1, n (\%) & $10(5.3)$ & - & 0.0586 \\
\hline ASA 2, n (\%) & $107(57.2)$ & $56(71.8)$ & \\
\hline ASA 3, n (\%) & $66(35.3)$ & $21(26.9)$ & \\
\hline ASA 4, n (\%) & $4(2.1)$ & $1(1.28)$ & \\
\hline \multicolumn{4}{|l|}{ Operative parameters: } \\
\hline Operative time, median (IQR) [min] & $180(150-240)$ & $180(160-235)$ & 0.4729 \\
\hline Intraoperative blood loss, median (IQR) [ml] & $50(50-150)$ & $100(50-200)$ & 0.0352 \\
\hline Colon, $n(\%)$ & $129(68.9)$ & $46(59.9)$ & 0.1198 \\
\hline Rectum, $n(\%)$ & $58(31.1)$ & $32(41.1)$ & \\
\hline \multicolumn{4}{|l|}{ Stage of cancer, $n(\%)$ : } \\
\hline AJCC stage I & $79(42.2)$ & $30(38.4)$ & 0.5327 \\
\hline AJCC stage II & $63(33.7)$ & $24(30.8)$ & \\
\hline AJCC stage III & $45(24.1)$ & $24(30.8)$ & \\
\hline \multicolumn{4}{|l|}{ Postoperative complications, $n$ (\%): } \\
\hline Clavien-Dindo I & & $35(13.2)$ & \\
\hline Clavien-Dindo II & & $17(6.4)$ & \\
\hline Clavien-Dindo III & & $19(7.2)$ & \\
\hline Clavien-Dindo IV & & $7(2.6)$ & \\
\hline
\end{tabular}

the ethical standards laid down in the 1964 Declaration of Helsinki and its later amendments.

\section{Statistical analysis}

All data were analyzed with StatSoft Statistica $v .13$. The results are presented as mean \pm standard deviation (SD), median and interquartile range (IQR) and hazard ratio (HR) with 95\% confidence intervals $(\mathrm{Cl})$ when appropriate. The study of categorical variables used the $\chi^{2}$ test of independence. The Shapiro-Wilk test was used to check for normal distribution of data and Student's t-test was used for normally distributed quantitative data. For non-normally distributed quantitative variables, the Mann-Whitney $U$ test was used. For the purposes of further analysis, the entire group of patients was divided into subgroups depending on occurrence of complications. Survival data were analyzed according to the Kaplan-Meier method. The log-rank test was used to detect differences between groups. Univariate and multivariate analysis was performed using Cox proportional hazards.

Variables for which $p<0.05$ were included in the model. Results were considered statistically significant when the $p$-value was found to be less than 0.05 . 


\section{Results}

When analyzing survival, we noted that patients without complications had significantly better overall 3-year survival compared to those with complications $(84.9 \%$ vs. $69.8 \%, p=0.022)$. Figure 1 shows Kaplan-Meier curves of overall survival according to presence of complications.

Patients without complications showed significantly improved survival rates compared with complicated patients, $p=0.00166$ (log-rank test for equality of survival functions).

Subsequent analysis of subgroups revealed that patients with severe complications (Clavien-Dindo III-IV) did not differ from those with less severe morbidity (Clavien-Dindo I-II) (Figure 2).

The Cox proportional multivariate model showed that postoperative complications $(\mathrm{HR}=2.83 ; 95 \% \mathrm{Cl}$ : 1.35-5.92; $p=0.0058)$ and AJCC III $(\mathrm{HR}=3.17 ; 95 \% \mathrm{Cl}$ : 1.52-6.6; $p=0.0021$ ) were independent predictors of worse survival after laparoscopic colorectal cancer surgery.

\section{Discussion}

This interim analysis of outcomes confirmed the association between postoperative complications and worse long-term overall survival after laparoscopic resection for non-metastatic bowel cancer. Patients who developed at least one complication had significantly lower 3-year survival compared to uncomplicated cases. Moreover, we did not note any difference in survival related to severity of postoperative complications - patients with Clavien-Dindo 1-2 complications had similar outcomes to those with Clavien-Dindo 3-4 complications.

More than 25 years after introduction to colorectal surgery the general attitude towards laparoscopy has evolved. In the past it was considered inferior to the open approach, leading to worse oncologic outcomes [18, 19]. However, the most recent analyses confirm the safety of laparoscopy, which is not inferior to open access surgery in terms of shortand long-term results $[20,21]$. In the conclusions of a systematic review comparing open and laparoscopic approach to colonic cancer, Di et al. stated that the apparent advantages of laparoscopy in short-term outcomes suggest that it should be widely recommended in clinical practice [20]. This could be attributed to the potential reduction in peri- and post-operative morbidity associated with minimal-

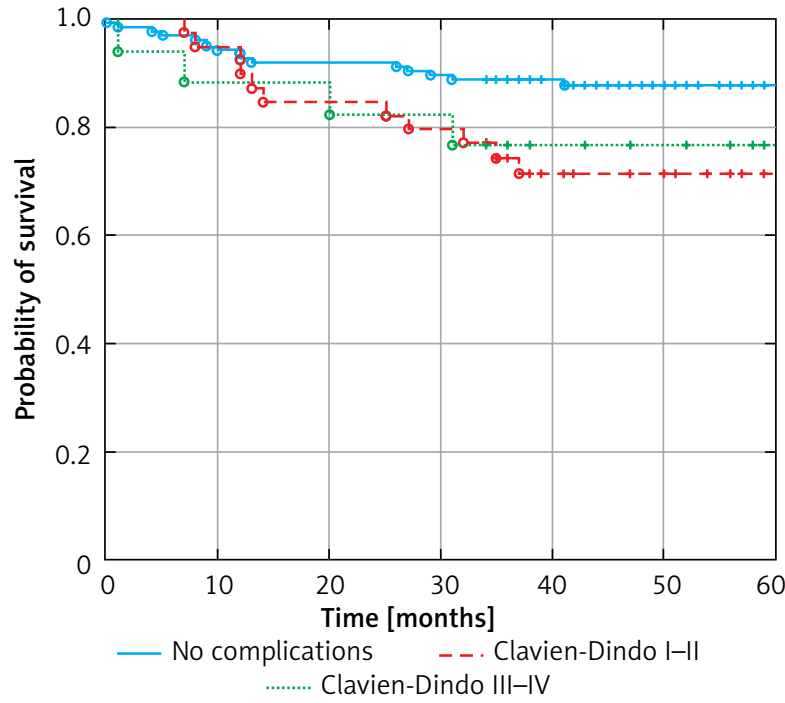

Figure 2. Kaplan-Meier curves of overall survival according to presence of complications and their severity (log-rank test forequality of survival functions)

ly invasive surgery. Interestingly, the most recently published randomized controlled trials covering this topic in rectal cancer surgery did not reveal differences in morbidity [21]. In contrast, registry analyses of large cohorts of patients clearly demonstrate benefits of minimally invasive access. Registries may be blamed for selection bias, but even randomized trials leave some space for criticism related to the specific limitations typical for this type of studies. On one hand they are prone to the Hawthorne effect, while on the other hand they usually show results of carefully selected high-volume centers, which may be different from the results at a national level. In addition, their inclusion criteria are often different from clinical characteristics of cancer patients in the general population.

The complication rate in our cohort was $29.4 \%$. This is in line with previously published reports [22-24]. Since 2013 the Enhanced Recovery After Surgery Protocol has been a standard of care in our institution and all patients included in the analysis were treated according to the ERAS protocol. This perioperative strategy is becoming the gold standard in care of patients submitted to surgery. Previous studies confirmed that application of ERAS shortens the length of stay and diminishes morbidity [25-27]. For instance, in colorectal surgery it has been shown to significantly improve outcomes by reduction of 
postoperative morbidity (mainly non-surgical) by up to $40 \%[28,29]$.

Careful analysis of cases showed significantly worse long-term survival after laparoscopic colorectal resection with complications Several factors might have contributed to this phenomenon. Firstly, complications postpone introduction of adjuvant chemotherapy. This is perhaps the strongest factor leading to inferior outcomes in group 2 (complicated). Several reports showed that delay in chemotherapy results in lower survival [30]. Moreover, even if chemotherapy was administered within the window of opportunity, patients with complications still presented with worse survival [30,31]. It has also been confirmed that chemotherapy after laparoscopic resection may be introduced earlier than after the open approach [30, 32, 33]. However, patients in our cohort had worse outcomes regardless of the severity of complications. Therefore, another explanation is needed requiring more profound studies of the role of infectious and inflammatory processes and the impact of the immune system influencing long-term survival among complicated patients [34-37]. Postoperative immunosuppression is certainly greater in patients with complications or after more severe surgical trauma (open surgery). Previous studies found a link between the immune response and cancer recurrence $[6,38-40]$. Another factor to include is the adrenal response to stress and infection [41, 42]. Both these factors have been shown to increase cortisol levels. There is evidence for an association between cortisol levels, stress, depression and development of metastases in other malignancies but a causative relationship still has not been documented $[43,44]$. All those factors - apart from delay in chemotherapymay have an influence on survival rates in patients with a complicated postoperative course.

Our study has several limitations. In this analysis we assessed only overall 3-year survival. For this reason, these are interim results rather than a full analysis of outcomes. The number of patients with complete 5-year observation was lower, so we decided to shorten the observation period in order to increase the number of individuals in the group and achieve meaningful results. We also measured only overall, not cancer-specific survival, which might also give biased results. When analyzing survival, we used the national personal identification number database (PESEL), which provides information on the date of death but not its cause. However, we believe that overall survival is the most important benchmark from the point of view of both clinicians and patients. Another limitation is the lack of precise data about type of adjuvant therapy regimens and treatment protocol compliance in our patients who received this chemotherapy in various institutions with potentially different standards of follow-up.

In our study colonic and rectal cases are discussed together, potentially leading to some bias due to different levels of complexity of surgery. However, it has been shown that in ERAS and laparoscopy environment, colonic and rectal resections provide similar outcomes in terms of short-term measures and morbidity [14]. Additionally, the composition of our cohort (approx. 40\% rectal cancer patients and $60 \%$ colon cancer patients) is similar to general epidemiology of colon and rectum cancer in western populations. Therefore, we would argue that our cohort was representative.

\section{Conclusions}

Postoperative complications after laparoscopic colorectal cancer surgery have a significant impact on long-term outcomes. For this reason, these patients should be carefully monitored after surgery, aiming at early detection of recurrence.

\section{Conflict of interest}

The authors declare no conflict of interest.

\section{References}

1. Hendren S, Birkmeyer JD, Yin H, et al. Surgical complications are associated with omission of chemotherapy for stage III colorectal cancer. Dis Colon Rectum 2010; 53: 1587-93.

2. Cohen ME, Bilimoria KY, Ko CY, Hall BL. Development of an American College of Surgeons National Surgery Quality Improvement Program: morbidity and mortality risk calculator for colorectal surgery. J Am Coll Surg 2009; 208: 1009-16.

3. Law WL, Poon JTC, Fan JKM, Lo OSH. Survival following laparoscopic versus open resection for colorectal cancer. Int J Colorectal Dis 2012; 27: 1077-85.

4. Sjo OH, Larsen S, Lunde OC, Nesbakken A. Short term outcome after emergency and elective surgery for colon cancer. Colorectal Dis 2009; 11: 733-9.

5. Kwiatkowski A, Stępińska G, Stanowski E, Paśnik K. Implementation of laparoscopic approach in colorectal surgery - a single center's experience. Videosurgery Miniinv 2018; 13: 27-32.

6. Wierdak M, Pisarska M, Kuśnierz-Cabala B, et al. Changes in plasma albumin levels in early detection of infectious complications after laparoscopic colorectal cancer surgery with ERAS protocol. Surg Endosc 2018; in press. 
7. Pędziwiatr $M$, Wierdak $M$, Nowakowski $M$, et al. Cost minimization analysis of laparoscopic surgery for colorectal cancer within the enhanced recovery after surgery (ERAS) protocol: a single-centre, case-matched study. Videosurgery Miniinv 2016; 11: 14-21.

8. Merkow RP, Bentrem DJ, Mulcahy MF, et al. Effect of postoperative complications on adjuvant chemotherapy use for stage II colon cancer. Ann Surg 2013; 258: 847-53.

9. Cheung WY, Neville BA, Earle CC. Etiology of delays in the initiation of adjuvant chemotherapy and their impact on outcomes for stage II and III rectal cancer. Dis Colon Rectum 2009; 52: 1054-63.

10. Poylin V, Curran T, Lee E, Nagle D. Laparoscopic colectomy decreases the time to administration of chemotherapy compared with open colectomy. Ann Surg Oncol 2014; 21: 3587-91.

11. Ptok H, Marusch F, Meyer F, et al. Impact of anastomotic leakage on oncological outcome after rectal cancer resection. $\mathrm{Br}$ J Surg 2007; 94: 1548-54.

12. Law WL, Choi HK, Lee YM, Ho JWC. The impact of postoperative complications on long-term outcomes following curative resection for colorectal cancer. Ann Surg Oncol 2007; 14: 2559-66.

13. Pędziwiatr M, Kisialeuski M, Wierdak M, et al. Early implementation of Enhanced Recovery After Surgery (ERAS ${ }^{\circledR}$ ) protocol compliance improves outcomes: a prospective cohort study. Int J Surg 2015; 21: 75-81.

14. Pędziwiatr M, Pisarska M, Kisielewski M, et al. ERAS protocol in laparoscopic surgery for colonic versus rectal carcinoma: are there differences in short-term outcomes? Med Oncol 2016; 33: 56.

15. Pisarska M, Pędziwiatr M, Małczak P, et al. Do we really need the full compliance with ERAS protocol in laparoscopic colorectal surgery? A prospective cohort study. Int J Surg 2016; 36: 377-82.

16. Dindo D. The Clavien-Dindo classification of surgical complications. Treat Postoper Complicat After Dig Surgery Springer, London 2014; 13-9.

17. Clavien PA, Barkun J, De Oliveira ML, et al. The Clavien-Dindo classification of surgical complications: five-year experience. Ann Surg 2009; 250: 187-96.

18. Berends FJ, Kazemier G, Bonjer HJ, Lange JF. Subcutaneous metastases after laparoscopic colectomy. Lancet 1994; 344: 58.

19. Wexner SD, Cohen SM. Port site metastases after laparoscopic colorectal surgery for cure of malignancy. Br J Surg 1995; 82: 295-8.

20. Di B, Li Y, Wei K, et al. Laparoscopic versus open surgery for colon cancer: a meta-analysis of 5-year follow-up outcomes. Surg Oncol 2013; 22: e39-43.

21. Pędziwiatr M, Małczak P, Mizera M, et al. There is no difference in outcome between laparoscopic and open surgery for rectal cancer: a systematic review and meta-analysis on short- and long-term oncologic outcomes. Tech Coloproctol 2017; 21: 595-604.

22. Loftus EV, Delgado DJ, Friedman HS, Sandborn WJ. Colectomy and the incidence of postsurgical complications among ulcerative colitis patients with private health insurance in the United States. Am J Gastroenterol 2008; 103: 1737-45.

23. Peyrin-Biroulet L, Germain A, Patel AS, Lindsay JO. Systematic review: outcomes and post-operative complications following colectomy for ulcerative colitis. Aliment Pharmacol Ther 2016; 44: 807-16.

24. Zogg CK, Najjar P, Diaz AJR, et al. Rethinking priorities: cost of complications after elective colectomy. Ann Surg 2016; 264 : 312-22.

25. Małczak P, Pisarska M, Piotr M, et al. Enhanced recovery after bariatric surgery: systematic review and meta-analysis. Obes Surg 2017; 27: 226-35.

26. Pisarska M, Małczak P, Major P, et al. Enhanced recovery after surgery protocol in oesophageal cancer surgery: systematic review and meta-analysis. PLoS One 2017; 12: e0174382.

27. Pedziwiatr M, Matłok M, Kisialeuski M, et al. Short hospital stays after laparoscopic gastric surgery under an enhanced recovery after surgery (ERAS) pathway: experience at a single center. Eur Surg - Acta Chir Austriaca 2014; 46: 128-32.

28. Kisialeuski M, Pędziwiatr M, Matłok M, et al. Enhanced recovery after colorectal surgery in elderly patients. Videosurgery Miniinv 2015; 10: 30-6.

29. Greco M, Capretti G, Beretta L, et al. Enhanced recovery program in colorectal surgery: a meta-analysis of randomized controlled trials. World J Surg 2014; 38: 1531-41.

30. Day AR, Middleton G, Smith RVP, et al. Time to adjuvant chemotherapy following colorectal cancer resection is associated with an improved survival. Colorectal Dis 2014; 16: 368-72.

31. Tevis SE, Kohlnhofer BM, Stringfield S, et al. Postoperative complications in patients with rectal cancer are associated with delays in chemotherapy that lead to worse disease-free and overall survival. Dis Colon Rectum 2013; 56: 1339-48.

32. Chau I. Adjuvant therapy in colon cancer: what, when and how? Ann Oncol 2006; 17: 1347-59.

33. Lohrisch C, Paltiel C, Gelmon K, et al. Impact on survival of time from definitive surgery to initiation of adjuvant chemotherapy for early-stage breast cancer. J Clin Oncol 2006; 24: 4888-94.

34. Mirnezami A, Mirnezami R, Chandrakumaran K, et al. Increased local recurrence and reduced survival from colorectal cancer following anastomotic leak: systematic review and meta-analysis. Ann Surg 2011; 253: 890-9.

35. Eberhardt JM, Kiran RP, Lavery IC. The impact of anastomotic leak and intra-abdominal abscess on cancer-related outcomes after resection for colorectal cancer: a case control study. Dis Colon Rectum 2009; 52: 380-6.

36. Lin JK, Yueh TC, Chang SC, et al. The influence of fecal diversion and anastomotic leakage on survival after resection of rectal cancer. J Gastrointest Surg 2011; 15: 2251-61.

37. Attiê R, Chinen LTD, Yoshioka EM, et al. Acute bacterial infection negatively impacts cancer specific survival of colorectal cancer patients. World J Gastroenterol 2014; 20: 13930-5.

38. Novitsky YW, Litwin DEM, Callery MP. The net immunologic advantage of laparoscopic surgery. Surg Endosc Other Interv Tech 2004; 18: 1411-9.

39. Ben-Eliyahu S. The promotion of tumor metastasis by surgery and stress: immunological basis and implications for psychoneuroimmunology. Brain Behav Immun 2003; 17 (1 Suppl.): S27-36.

40. Evans C, Galustian C, Kumar D, et al. Impact of surgery on immunologic function: comparison between minimally invasive techniques and conventional laparotomy for surgical resection of colorectal tumors. Am J Surg 2009; 197: 238-45. 
41. Torpy DJ, Ho JT. Value of free cortisol measurement in systemic infection. Horm Metab Res 2007; 39: 439-44.

42. Het S, Schoofs D, Rohleder N, Wolf OT. Stress-induced cortisol level elevations are associated with reduced negative affect after stress: indications for a mood-buffering cortisol effect. Psychosom Med 2012; 74: 23-32.

43. Sephton SE, Dhabhar FS, Keuroghlian AS, et al. Depression, cortisol, and suppressed cell-mediated immunity in metastatic breast cancer. Brain Behav Immun 2009; 23: 1148-55.

44. Matsuo K, Prather CP, Ahn EH, et al. Significance of perioperative infection in survival of patients with ovarian cancer. Int J Gynecol Cancer 2012; 22: 245-53.

Received: 9.05.2018, accepted: 22.05.2018. 